\title{
Le virus en territoire, entre perceptions et mesures
}

\section{Sandra Pérez, Nicole Vernazza-Licht et Daniel Bley}

\section{(2) OpenEdition}

\section{Journals}

Édition électronique

URL : http://journals.openedition.org/rfst/875

DOI : $10.4000 /$ rfst.875

ISSN : 2492-3672

Éditeur

Espaces et SOciétés (UMR 6590)

Référence électronique

Sandra Pérez, Nicole Vernazza-Licht et Daniel Bley, « Le virus en territoire, entre perceptions et mesures ", Revue francophone sur la santé et les territoires [En ligne], Pandémie, crises et perspectives lectures territoriales de la Covid-19, mis en ligne le 11 mars 2021, consulté le 06 avril 2021. URL : http://journals.openedition.org/rfst/875 ; DOl : https://doi.org/10.4000/rfst.875

Ce document a été généré automatiquement le 6 avril 2021.

La Revue francophone sur la santé et les territoires est mise à disposition selon les termes de la Licence Creative Commons Attribution - Pas d'Utilisation Commerciale - Partage dans les Mêmes Conditions 4.0 International. 


\title{
Le virus en territoire, entre perceptions et mesures
}

\author{
Sandra Pérez, Nicole Vernazza-Licht et Daniel Bley
}

\section{NOTE DE L'AUTEUR}

Les auteurs appartiennent au groupe de travail « Espace au corps » de l'UMR ESPACE

\section{Introduction}

1 La maladie Covid-19 sévit sur l'ensemble de la planète ${ }^{1}$ et il est difficile de présumer de l'évolution de l'épidémie sur le territoire français après plus d'un semestre ${ }^{2}$. Néanmoins dans ce contexte d'incertitude nous pouvons dès à présent, nous interroger sur le lien entre gestion d'une épidémie infectieuse émergente, inconnue et évolutive et l'application du principe de précaution au plan national pour la mise en place de mesures de santé publique de prévention et de soins.

Le principe de précaution repose sur l'anticipation dans un contexte de connaissances incertaines d'un ou plusieurs effets produits par un ou plusieurs évènement(s), ou substance(s) qui porterai(en)t atteinte à l'intégrité physique des personnes. Les actions prises dans ce cadre ont pour objectif l'atténuation, voire dans le meilleur des cas la suppression $\mathrm{du}$ risque, alors que les enjeux sont forts (nombreuses victimes ou personnes potentiellement vulnérables). «This principle provides a moral justification for acting even though causation is unclear » (Ricci et al., 2011), c'est là tout son intérêt, et sa force, même si le résultat est lui-même incertain : "Precautionary principles are the foundations for policy when it has to deal with weakly understood causes of potential catastrophic or irreversible events, and where protective decisions require certain and costly policy interventions that may not solve the problem that they are designed to correct » (Ricci et al., 2013). 
Cependant, dans sa force réside également sa faiblesse, car parfois, le principe de précaution ne s'applique pas sans dommages collatéraux « fundamentally, the dilemma is that the ethical choice, better safe than sorry, can be costly because an action designed to avoid a potential damage can be counterproductive for society : a seemingly precautionary action can do more harm than good» (Renn, 2015). En fait, le principe de précaution devrait être proportionné, c'est-à-dire que le coût des actions de prévention ne doit pas être disproportionné par rapport aux bénéfices attendus, « Proportionality principle, in which the costs of actions to prevent or minimize hazards should not be disproportionate to the likely benefits » (T.M.C. ASSER INSTITUUT, 2011).

4 Ainsi, il nous parait intéressant de réfléchir au décalage qui peut exister entre une connaissance objectivée du virus détenue par des scientifiques, ou des experts, les mesures de santé publique qui vont en découler et la perception de cette connaissance par les habitants, au sein de leur territoire. Que se passe-t-il, en fait à la rencontre du top down et du bottom up ? Comment s'articulent mesures de l'espace, principe de précaution, mesures de prévention, et perceptions de la population.

Une distance existe souvent entre les perceptions des populations sur une question donnée, et l'avis des experts fondé sur des données scientifiques. Cette distance est parfois renforcée par la méconnaissance de la part de la population des méthodes employées par les épidémiologistes, et par le fait que les scientifiques ne sont pas in situ alors que la population peut se prévaloir de mieux connaitre les dimensions locales. Les études psycho-sociologiques ont mis en évidence que le risque est surestimé lorsqu'il est subi (sentiment de non maîtrise), par rapport à un risque « choisi " (mode de vie). Si le risque est évalué de manière globale, populationnelle, par les organismes (par exemple : 1 chance sur 1 million), la potentialité de devenir soi-même une victime rendrait toute exposition inacceptable (Setbon, 2004).

Par ailleurs, cette perception évolue selon les fonctions de chacun, la connaissance (Pérez et al., 2020), l'intérêt que l'on peut avoir pour un sujet ${ }^{3}$, et donc bien entendu avec le temps, qui apparaît comme une dimension essentielle dans un contexte épidémique.

7 De plus, alors que les institutions publiques ont en charge le collectif, la population répond souvent par de l'individuel. Se pose alors le problème pour le conseil scientifique Covid-194 de la variabilité interindividuelle alors qu'il est à la recherche d'invariants (adoption au même niveau des mesures barrières sur l'ensemble du territoire). De ce fait, en situation de crise, d'urgence, d'incertitude, la tentation est grande de prendre des actions uniformes sur l'ensemble du territoire même si à un niveau infra la situation apparait beaucoup plus nuancée.

8 Parfois, la prévention passe à côté de sa "cible", les individus ne prennent pas conscience d'un risque, car ils sont trop préoccupés par leurs conditions matérielles à court terme. Ainsi, ce ne sont pas forcément les personnes qui sont le plus exposées qui sont le plus conscientes d'un risque qu'il soit environnemental ou de santé, et si elles le sont, elles peuvent alors être tentées par une forme de déni (Pérez, 2019).

9 De plus, les populations sont à l'heure actuelle plus sensibilisées, plus (mais pas forcément mieux) informées, sur les questions relatives à leur santé. Mais cette connaissance "profane", qui la plupart du temps provient des médias peut être tronquée, partielle, voire partisane, et susciter de la peur. 
10 La compréhension de la perception du risque par la population est essentielle pour faire passer les messages de prévention les plus ad hoc, et lutter ainsi contre les fake news. Signalons à ce propos le développement du fact checking, où les réseaux sociaux mettent en place des procédures pour lutter contre les fake news concernant la Covid-19, et ce d'autant plus que l'impact des fake news semble plus important, et leur contenu plus facilement assimilable que celui d'une vérité mesurée.

11 Nous allons présenter dans cet article la mise en application du principe de précaution dans la gestion de l'épidémie de la Covid- $19^{5}$ à partir notamment de la prise en charge qui a été adoptée pour éviter des contaminations professionnelles et familiales postmortem. Nous allons voir sur quelle base de connaissances il a été appliqué, et comment il a été vécu par les populations directement concernées. Il s'agit d'un bon exemple pour réfléchir sur les perceptions des populations et les mesures de santé publique.

12 Puis, nous montrerons comment ces différences mesures/perceptions peuvent se traduire dans la gestion de l'épidémie selon le choix qui est fait d'une politique étendue à l'ensemble du territoire, ou d'une décentralisation des décisions au niveau régional.

13 S'agissant des sources de données utilisées dans cet article il faut souligner que les données scientifiques publiées sur ces questions, notamment dans le domaine des SHS, sont encore peu nombreuses ou pas toujours validées par des pairs, ce qui a rendu l'analyse plus difficile.

14 C'est aussi la raison pour laquelle dans la mesure où la question de cette épidémie a quasiment occupée la presque totalité de l'espace public, nous avons utilisé, outre les articles scientifiques, des données issues de la littérature grise qui ont pu nous renseigner sur les perceptions de la population et les degrés d'implication de la population et des différents acteurs (cf. en note la liste des sites et documents consultés ${ }^{6}$. Nous nous sommes aussi appuyés sur les avis, rapports et communiqués officiels des différentes institutions (HAS, HCSP, Académie de Médecine, Conseil scientifique COVID-19, Ministère de la Santé, OMS...).

$15 \mathrm{Au}$ niveau de la méthode, nous avons conjugué la recension et l'analyse des sources de données précédemment citées avec une approche qualitative. Nous avons eu également des entretiens avec des professionnels de santé (épidémiologistes, médecins de santé publique, médecins généralistes, pharmaciens) et des professionnels du domaine du funéraires.

\section{Épidémie émergente et évolution des connaissances scientifiques : l'exemple de la gestion du deuil}

16 Dans la dernière semaine du mois de mars 2020, le Ministre de la Santé, Olivier Véran annonçait qu'une personne décédait toutes les 4 minutes de la Covid-19 en France. La question de la contagiosité du corps des défunts se posait depuis le début de l'épidémie avec acuité en France, comme dans le reste du monde, et la Direction Générale de la Santé (DGS) avait sollicité l'avis du Haut Conseil de la Santé Publique ${ }^{7}$ (HCSP).

17 Au mois de mai 2020 un article scientifique est publié dans le Journal of Forensic and Legal Medecine relatant un cas de contamination post-mortem d'un médecin légiste décédé en Thaïlande après avoir été en contact avec des défunts infectés par la Covid-19 (Sriwijitalai et al., 2020). Cet article a ensuite fait l'objet d'un rectificatif ${ }^{8}$, car il n'était 
pas possible de savoir si la victime avait été contaminée sur le lieu de son travail, ou bien dans d'autres espaces.

Début février, le HCSP dans son Avis sur «la prise en charge du corps d'un patient décédé » avait mentionné la survie très allongée de la plupart des agents infectieux et la nécessité d'appliquer des précautions lors de la manipulation des corps (HCSP, 18 février 2020). Pour éviter toute transmission du virus, le HCSP préconisait donc de ne réaliser aucun acte de thanatopraxie (soins de conservation) sur les corps de patients décédés de la Covid-19 et invitait les personnels de santé à placer le corps dans une housse imperméable dès le constat du décès. En effet, "La manipulation d'un corps peut exposer le personnel le manipulant à des germes à transmission aérienne". Des mesures étaient prises afin de limiter les risques de contamination post-mortem. Transféré en chambre mortuaire, housse fermée, le corps est déposé en cercueil simple et la mise en bière doit être immédiate comme le précise la recommandation "qu'il soit procédé sans délai à la fermeture définitive du cercueil ».

19 Ces mesures vont créer des situations de tensions notamment de la part des familles endeuillées, mais aussi parmi les travailleurs du funéraires et les institutions religieuses concernées, situations que les parlementaires, en particulier, vont prendre en considération à partir de deux groupes de travail en expliquant «nos activités d'élus nous ont amenés à recevoir les doléances de personnes qui n'avaient pu accéder à leurs proches dans les derniers moments de leur vie, ni même réaliser de cérémonie funéraire... » (OPECST, 2020a et b). Elles seront qualifiées par la suite, par le Groupe de Travail du HCSP, de "mesures maximalistes » en l'état des connaissances disponibles en ce début de prise en charge de l'épidémie en France (HCSP, avis du 24 mars 2020 : 1).

Cet avis sera remplacé un mois plus tard suite à une saisine de la DGS qui demandait de mettre en adéquation le premier avis avec les nouvelles connaissances dans les avis rendus entre-temps sur la prise en charge des patients, et de détailler plus largement les modalités de gestion des corps pour les professionnels du funéraires9.

21 Le HSCP déclare alors que "Le risque infectieux ne disparait pas immédiatement avec le décès d'un patient infecté, mais les voies de transmission sont réduites, et en particulier la voie respiratoire, qui constitue le mode principal de transmission du SARS-CoV-2". Par ailleurs, le virus a été retrouvé dans les voies aériennes supérieures et profondes de défunts, ainsi que dans leur système digestif (HCSP, 24/03/2020, p.1). Les précautions recommandées pour la protection du personnel de santé comme pour le personnel funéraire sont largement détaillées selon le contexte du décès (hôpital, EPHAD, domicile).

Contrairement au premier avis du HCSP, publié le 18 février, celui du 24 mars 2020 autorise à nouveau un soin du corps du défunt : "il convient de respecter la stricte observance des règles d'hygiène et de mesures de distance physique, ...notamment en ce qui concerne la toilette rituelle $d u$ corps par les personnes désignées par les proches, ainsi que la possibilité pour ceux-ci de voir le visage de la personne décédée avant la fermeture définitive $d u$ cercueil". Les contacts sont restreints, les mesures barrières appliquées. Le personnel en charge de la toilette mortuaire, de l'habillage ou du transfert dans une housse est équipé d'une tenue de protection adaptée (lunettes, masque chirurgical, tablier antiprojection, gants à usage unique). Concernant les effets personnels du défunt: ils doivent être lavés à plus de 60 degrés pendant au moins 30 minutes ou désinfectés. Sinon, ils sont mis dans un sac plastique fermé pendant 10 jours. 

patients décédés, et en particulier quant à l'attitude à adopter vis-à-vis de la famille dans la mesure où il est précisé que "l'infection par le SARS-CoV-2 n'est pas considérée comme relevant d'une mise en bière immédiate ${ }^{10} »$ (HCSP, mars 2020 p.2). Les familles ont ainsi du temps pour voir le corps du défunt. Le HCSP recommande ainsi le maintien d'une ouverture de la housse permettant à la famille de voir le visage en chambre hospitalière, mortuaire ou funéraire. Les rituels religieux peuvent être pratiqués par deux personnes maximum avec le même équipement que le personnel hospitalier ou funéraire afin d'éviter tout risque de contamination.

Cet avis constitue une avancée dans la mesure où il accorde une place centrale aux principes d'humanité qui doivent régir les relations autour d'une personne décédée. Dans cet avis, le HCSP tient compte des dimensions éthiques et anthropologiques qui ne figuraient pas dans l'avis précédent et qui sont clairement mentionnées en préambule de ses recommandations où il rappelle qu'il convient... « de respecter dans leur diversité les pratiques culturelles et sociales autour du corps d'une personne décédée » (HCSP, mars 2020, p.2).

tient compte aussi de l'expérience acquise, avec l'appui en particulier des chercheurs en Sciences Humaines et Sociales lors des récentes épidémies d'Ebola, et notamment sur l'impérieuse nécessité pour les familles et leur entourage de donner une visibilité aux malades et aux personnes décédées, de pouvoir ritualiser ces moments et ce, que ce soit dans les sociétés africaines ou européennes (Epelboin, 2020 ; Cros, 2015 ; GasquetBlanchard, 2015, 2016). En effet, si les mesures sont trop fortes et/ou sont perçues comme disproportionnées nous risquons alors de nous retrouver dans la situation évoquée par Clélia Gasquet (2015) où «les processus politiques mis en place pour prévenir l'émergence de la maladie [Ebola] puis son endiguement (suivi des sujets contacts, isolement des malades, inhumations par les équipes soignantes, etc.), apparaissent comme les apanages des dominants (politiques, médecins des pays du Nord). Il est alors fréquent d'observer que localement, les populations élaborent des processus, d'adoption, d'acceptation ou de rejet des stratégies internationales mises en œuvre contre la maladie. » Rejet qui est à éviter, tant la lutte contre l'épidémie passe par l'adoption et le respect des gestes barrières.

\section{Les perceptions des différents acteurs autour de la gestion des corps}

Ces nouvelles dispositions énoncées dans l'avis du 24 mars devaient convenir aux familles qui peuvent ainsi «faire leur deuil $»^{11}$ dans un contexte difficile puisque les cérémonies d'obsèques sont supprimées afin d'éviter tous risques liés aux rassemblements. Elles sont de nature à satisfaire également le personnel de nombre d'établissements de santé qui avaient également manifesté leur souhait de laisser les familles se recueillir au pied du lit du défunt.

Par contre, le personnel des entreprises du funéraires qui est insuffisamment équipé (pénurie de masques et de gants en particulier ${ }^{12}$ ) peut craindre une contamination et exercer son droit de retrait. Du point de vue des praticiens, en l'occurrence ici les thanatopracteurs les avis divergeaient concernant l'appréciation du risque. Certains craignaient de devoir réaliser des ponctions pulmonaires afin de vérifier si le défunt

Revue francophone sur la santé et les territoires, Pandémie, crises et perspectives : lectures territoriales de la Covid-19 
était bien décédé du virus, ce qui aurait eu comme conséquence de potentiellement répandre le virus dans les locaux, tandis que d'autres rappelaient que les produits utilisés lors des soins mortuaires contiennent suffisamment de virucides, bactéricides et fongicides pour rendre le virus inopérant.

Par ailleurs l'augmentation des décès dans certaines régions (le Grand Est, les Hauts de France et l'Ile de France), dont on peut craindre alors qu'elle devienne exponentielle provoque des difficultés de gestion. Dans beaucoup de pays, compte tenu du nombre de morts et de l'urgence sanitaire, des cercueils sont entassés dans des espaces libres réquisitionnés (cf. par exemple en France, le Hall du marché de Rungis) ${ }^{13}$ voire enterrés dans des fosses communes comme aux USA ainsi que le montreront des reportages télévisés ${ }^{14}$. S'agissant souvent d'un passage en boucle sur des chaines d'information continue ces images et les propos diffusés participent souvent de réactions émotionnelles exacerbées.

Toutefois, dans un décret du $1^{\text {er }}$ avril paru le 2 avril 2020 au Journal officiel (p. 9), le gouvernement ne suit pas l'avis des experts du HCSP et interdit la toilette mortuaire, les soins de conservation et exige la mise en bière immédiate des corps. Cette décision va engendrer un grand désarroi dans les familles qui vont alors être dans l'impossibilité de dire un ultime adieu à leur proche. Sa justification ne semble pas médicale, même si nous sommes en plein pic épidémique avec une saturation des établissements de soins, mais elle est plutôt due à une volonté de protection du personnel funéraire dont les représentants ont fait valoir leur point de vue auprès des autorités politiques.

Ce décret vise donc dans l'urgence et à court terme, à calmer les appréhensions des acteurs du funéraires. Cette décision qui parait légitime pour les décideurs afin de répondre aux risques encourus par les personnels, est toutefois susceptible de générer à long terme de véritables traumatismes psychologiques individuels, familiaux et sociaux et par conséquent de renforcer la fragilisation liée au vécu de la pandémie Covid-19.

31 Différents témoignages et articles journalistiques et scientifiques rendent alors compte de ces difficultés. L'entretien qu'accorde la sociologue et anthropologue Pascale Trompette spécialiste des questions liées à la mort et au funéraire est illustratif à plusieurs titres (Lecompte, 2020). Elle montre que les conditions qui entourent l'organisation des obsèques sont très éprouvantes pour les familles et parfois anxiogènes pour les employés du funéraires. C'est lié en premier lieu au pic de mortalité et en particulier au fait que les décès concernent des personnes âgées mais aussi plus jeunes, souvent autour de la cinquantaine, qui décèdent rapidement. Elles tiennent également à l'impossibilité pour les familles comme nous l'avons déjà évoqué de ne pas voir le défunt « On voit un proche partir à l'hôpital, et quelques jours plus tard, on se retrouve face à un cercueil fermé. Dans la perspective du deuil, c'est une épreuve très difficile et les familles demandent parfois aux pompes funèbres de photographier leur défunt.". D'autres essaient de se consoler en envisageant de reporter une cérémonie familiale après l'épidémie. Les employés du funéraires, quant à eux, sont pris dans une tension entre deux tendances contradictoires, tenant d'une part à sécuriser leurs gestes en contexte de Covid-19 et les peurs que le risque quotidien génère chez eux, problème psychologique parfois accru par le fait « de se retrouver totalement seuls au cimetière pour procéder à l'inhumation, sans aucun proche du défunt ». Les relations avec les familles se déshumanisent, se limitant souvent au seul contact téléphonique et les gestes d'accompagnement qui participent de leur activité ne peuvent être réalisés. 
Le même constat est fait par les chercheurs qui se sont intéressés, dans le contexte de la Covid-19, aux professionnels de santé en milieu hospitalier. Ils montrent, une difficulté plus grande liée au fait de ne pas pouvoir préparer les familles au décès, et lors du décès ne pas les laisser accéder au corps du défunt ou avoir un accès plus restreint, de ne pas pouvoir réaliser les toilettes mortuaires (Gueullette, 2008 et Hernandez). Ces pratiques et habitudes professionnelles sont parfois aménagées en fonction des services comme par exemple à l'Hôpital européen de Marseille (Kra et ali, 2020).

Nous pouvons notamment nous appuyer sur la note produite par l'office parlementaire d'évaluation des choix scientifiques et technologiques sur «la crise du funéraires en situation de Covid-19» qui a auditionné des chercheurs (historien, philosophe, sociologues et anthropologues) ainsi que des représentants d'entreprises du funéraires. Les parlementaires font état des doléances qu'ils ont reçu de la part des personnes endeuillées et meurtries, mais également de la situation des professionnels du funéraires, travailleurs qualifiés "d'invisibles ", en manque de reconnaissance sociale ${ }^{15}$ malgré le maintien de leurs activités, pendant le confinement notamment. Ils notent les difficultés liées à l'application de ce décret en indiquant qu'ils ont $\mathrm{pu}$ « ... constater que tous les acteurs $d u$ mourir - soignants, familles endeuillées, professionnels du funéraire, représentants des pouvoirs publics - ont été durement éprouvés par les restrictions encadrant le milieu du funéraire depuis le début de la crise sanitaire » (OPECST, 2020a).

En effet, du point de vue des autorités, le rassemblement même limité de plusieurs personnes venant de diverses régions du pays pouvait légitimement contribuer à la création de nouveaux « clusters ${ }^{16}$, et ce, d'autant plus que dans de telles circonstances le respect des gestes barrières est difficile tant on peut avoir envie de manifester physiquement son soutien à la famille endeuillée par des embrassades ou le serrage de mains traditionnels.

35 Nous voyons bien à travers ces quelques éléments que cette situation de crise a été difficile à gérer, entre le point de vue des experts, les perceptions et le vécu des populations, les doléances des professionnels et les décisions des politiques.

La figure ci-dessous synthétise les différentes étapes dans la gestion de la crise que nous venons d'évoquer, elle rend compte également de l'impact des décisions prises sur les perceptions des acteurs concernés. 


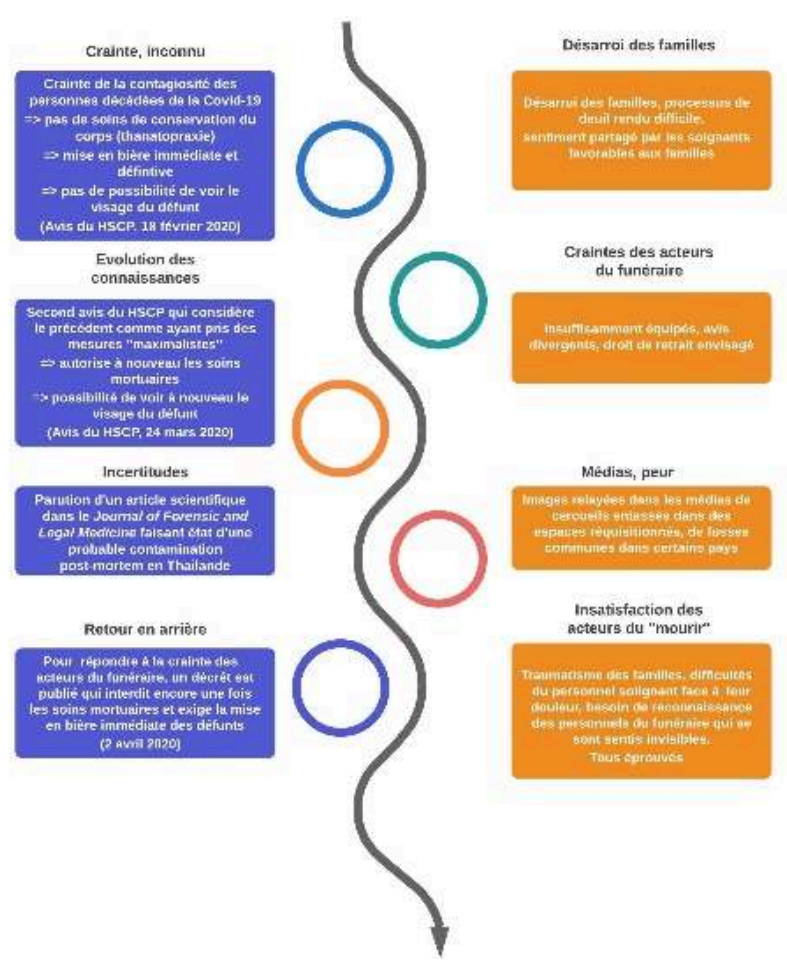

\section{Le décalage entre mesures de santé publique et perceptions territoriales du virus}

A partir de l'exemple de la gestion du corps des personnes décédées de la Covid-19 nous pouvons considérer que la mise en application du principe de précaution dans une politique de santé publique est dépendante de la perception du risque par les populations. Ce qui revient à dire que le principe de précaution doit apparaitre comme une réponse proportionnée afin de pouvoir être admise par la population, «openness and transparency are required as is adherence to the democratic principle that the consent of the governed is required when great changes are made » (OCDE, 2020).

Dans leur note sur « La crise du funéraires en situation de Covid-19 » les parlementaires considèrent que "la mise en cuvre de directives nationales, et de leur compréhension au niveau local» peut "provoquer des inégalités territoriales» (OPECST, 2020a: 9). C'est probablement le cas du décret du 2 avril qui met en place des directives nationales alors que la situation et l'évolution de l'épidémie de la Covid-19 sont très diversifiées sur le plan local. En effet, dès le début de l'épidémie la France va être divisée en zones qui évolueront au fur et à mesure de l'expansion du virus sur le territoire, puis en fonction du nombre de personnes contaminées ou en réanimation. Le Ministre de la Santé parle ainsi de "Carte mosaïque " pour expliquer les différences régionales le 4 mars 2020. Au début de l'épidémie il y aura quatre zones correspondant à 4 stades de circulation du virus. Par la suite, pour mieux communiquer et visualiser les différences, les zones seront également différenciées par des couleurs.

Les statistiques de mortalité, d'hospitalisation et de réanimation rendent compte au jour le jour de cette situation différentielle en mettant en évidence les zones (Grand Est, Haut de France et Ile de France) de fortes tensions et de concentration des cas alors 
que nombre de régions ne sont pas ou faiblement concernées (Levratto et al. 2020 ${ }^{17}$; Grimault, 2020). Dans la mesure où les situations épidémiologiques sont différentes selon les régions, un traitement national uniforme pour les mesures mises en place, qui ne prend pas en compte ces différences, n'est pas équitable à défaut d'être réellement inégalitaire, et il sera perçu comme tel. Sur l'ensemble du territoire français le décret du 2 avril prive néanmoins nombre de familles de la possibilité de voir le corps de leur défunt, de l'enterrer dans le respect de mesures sanitaires dites «barrières ». Il ne peut dès lors susciter que de l'incompréhension devant des mesures décidées depuis la capitale, alors que la population en région a un vécu quotidien et une perception différente de l'impact et de la circulation du virus, plus conforme d'ailleurs à l'hétérogénéité spatiale ${ }^{18}$ de l'épidémie au cours du printemps 2020.

40 La fermeture des frontières pour limiter la circulation du virus en réduisant les mobilités extérieures, et le confinement généralisé de la population française au printemps 2020, alors que l'épidémie est concentrée à quelques régions, puis, et surtout la phase de déconfinement avec la mise en images de l'hexagone français en trois couleurs (verte, orange, rouge) vont accentuer l'incompréhension dans la population. Les différentes mesures politiques privilégiées pour gérer la crise sanitaire vont faire resurgir ou créer des clivages identitaires territoriaux Paris/régions. Un décalage entre les perceptions territoriales profanes de la circulation du virus, les données du Ministère de la Santé, et les mesures de santé publique va être mis en évidence. Ces différents épisodes de la lutte contre la Covid-19 vont susciter également de la colère, des tensions, voire de l'opposition contre la politique menée. Le terme de jacobinisme ${ }^{19}$ sera largement utilisé à de nombreuses reprises pour qualifier les mesures adoptées dans la lutte contre l'épidémie. Ainsi l'économiste de la santé Pierre-Yves Geoffard intitule-t-il son éditorial dans le journal des Echos en septembre $2020^{20}$ : «Covid-19 : les limites du jacobinisme sanitaire » pour expliquer que la gestion sanitaire au printemps 2020 s'est révélée « rigide et centralisée ».

41 C'est également l'analyse du géographe Laurent Chalard ${ }^{21}$ qui considère par contre que la référence au jacobinisme par les élus du sud de la France est dû à un sentiment de stigmatisation qui est principalement le reflet de l'accentuation des fractures territoriales au sein de l'hexagone. Martin Vanier, urbaniste, dans un article qu'il a intitulé sur une forme interrogative "République des territoires à déconfiner?» considère également que la crise de la Covid-19 interroge la décentralisation, mais il est plus négatif quant à l'attitude des élus locaux et voit par contre dans cette expérience de gestion sanitaire que provoque la pandémie, l'opportunité de travailler à la société de l'efficacité globale.

Un autre exemple emblématique qui met en évidence la question de la territorialisation autour de la Covid-19 est celui du conflit entre le Pr. Didier Raoult et le Conseil scientifique sur la gestion des malades, de leur dépistage et de l'utilisation de la chloroquine pour les personnes positives. Ce qui n'aurait dû être qu'un débat d'experts sur les données et les méthodes de gestion a rapidement mis en exergue un clivage territorial et scientifique compte tenu de la reconnaissance scientifique internationale $\mathrm{du}$ Professeur Didier Raoult par rapport à ses contradicteurs, essentiellement infectiologues parisiens. Les différentes prises de position, de politiques, de scientifiques et de médecins et même d'organisations comme par exemple le soutien de l'ordre des médecins de la région $\operatorname{Sud}^{22}$ ont tendu à formaliser un discours de stigmatisation vis-à-vis du sud-est de la France en mettant en avant des différences 
régionales. Les médias, tout en montrant les contradictions, ont accentué ces différences de perceptions territoriales en les mettant en scène. La population marseillaise et plus largement de la région Sud, même si elle n'est pas la seule, s'est majoritairement reconnue dans ce discours identitaire et $\mathrm{y}$ a "affectivement" adhéré23.

Ce différend, pose aussi la question de l'expert dans l'arène publique, de la multitude d'infectiologues dans les médias, du manque de professionnalisme dans la communication, des nuances et imprécisions dans les données, ce qui a concouru à un manque de clarté sur les données scientifiques dont nous disposions et dans les décisions prises ou à prendre. En remettant en lumière la rivalité traditionnelle Paris/ Marseille, ce conflit s'est cristallisé autour de perceptions identitaires. Il a mis en évidence l'ancrage territorial des positions, mais aussi dans la population les perceptions différentielles du virus, et de son impact selon les situations locales.

Nous voyons donc bien que la gestion du risque est à considérer sous l'angle de la territorialisation et qu'en France la centralisation (c'est-à-dire le peu d'autonomie laissé aux régions) n'est pas forcément l'approche la plus appropriée pour la mise en place d'une politique de santé publique, surtout qu'elle n'emporte pas l'adhésion des populations qui en dernier ressort est le meilleur garant de la réussite de mesures de santé publique. Cependant, dans un pays où l'Etat est perçu comme un Etat Providence, protecteur, et où l'égalité est le ferment des institutions, nous comprenons bien que les inégalités de traitement qui seraient perçues par la population suivant leur territoire d'appartenance seraient en la matière, peut-être plus qu'en toute autre, très mal vécues ${ }^{24}$. D'où le difficile équilibre à trouver entre ces deux positions.

L'orientation de la politique sanitaire d'urgence contre le Covid-19 a été de privilégier des décisions nationales qui probablement n'ont pas prises en compte des réalités régionales qui pouvaient et étaient différentes. Ces décisions étaient pensées selon des principes égalitaires en termes d'espace national ${ }^{25}$. Le risque potentiel lié aux mobilités inter-régionales et la volonté de créer des barrières à la pandémie a peut-être pesé dans le choix de décisions appliquées au plan national. La mise en place de ces décisions a posé des problèmes qui montrent les avantages et les inconvénients de la territorialisation, et notamment, parmi ceux-ci, les difficultés liées à la multiplicité des acteurs (Préfets, Agences Régionales de Santé, collectivités locales) peu habitués à travailler ensemble.

Il faut toutefois souligner que les experts et les politiques ont, avec un peu plus de recul sur l'expansion du virus et une amélioration des connaissances, revu leurs positions et recommandations. Ainsi, nous observons à la fin de l'été 2020 une logique un peu différente, et des tentatives de mise en place de mesures au plan local (port du masque dans certaines villes, tendant toutefois vers une généralisation) ${ }^{26}$.

Des tentatives locales de gestion du risque Covid-19 peuvent être soulignées à titre d'exemples comme celles émanant d'élus Corses en matière de dépistage des voyageurs lors de l'été 2020, puis des fêtes de fin d'année, ou la mise en place par des élus municipaux de campagnes de dépistage généralisées alors que les recommandations nationales les limitaient aux personnes ayant des symptômes ou aux cas contact.

Les mesures au deuxième semestre 2020 restent néanmoins centralisées tout en affichant une volonté de territorialiser (usage des masques, taille des rassemblements, confinement partiel envisagé etc...). 


\section{Conclusion} les conséquences sociales et humaines à long terme de décisions trop globales. En effet, dans l'organisation de sa politique vis-à-vis du Covid-19, l'Etat a eu du mal à adopter des logiques consensuelles et qui tiennent compte des diversités régionales. La gestion des corps des personnes décédées en est un bon exemple, puisqu'en choisissant de mener une politique avec un décret national applicable en tous lieux alors que le risque viral n'était pas uniforme sur tout le territoire et son extension future mal connue, la diversité des populations concernées (familles et professionnels de santé et du funéraires), mais surtout la souffrance qui pouvait en résulter à court et long terme n'a pas été prise en compte.

51 A ce stade, l'épidémie de la Covid-19 aura mis en évidence les difficultés des experts et des décideurs à la fois séparément, mais également dans leurs interactions. Les experts étant enfermés dans des logiques de pensée, ayant du mal à produire des avis dans un contexte d'incertitude et d'évolution rapide des connaissances scientifiques (pas toujours d'accord entre eux, ne sachant pas communiquer), tandis que les politiques «naviguent à vue» entre avis des experts, intérêts particuliers (funéraires, professionnels de santé, etc...) et les réactions des populations. Elle aura aussi montré la nécessité de prendre en compte les perceptions des populations, entre peurs, sentiment de traitement inégalitaire, besoin d'autonomie, afin de produire des messages de prévention auxquels elles puissent adhérer.

Même si on doit prendre en considération le contexte d'urgence sanitaire, il nous semble, autour de l'exemple de la gestion du corps des personnes décédées qu'une politique plus décentralisée, prenant en compte les réalités locales et les incertitudes sur l'évolution du virus eut été mieux perçue et acceptée, voire peut être plus efficace.

Nota Bene : Le 30/11/2020 le HCSP a rendu un nouvel avis à la demande de la DGS afin d'actualiser ses recommandations au regard de l'évolution des connaissances sur le virus. Cet avis sur « la Prise en charge du corps d'une personne décédée et infectée par le coronavirus SARS-CoV-2 : actualisation des recommandations » ne préconise pas la « mise en bière immédiate » car, dixit, l'impossibilité de voir le corps du défunt dans un délai de $24 \mathrm{~h}$ pourrait avoir de graves conséquences psycho-sociales sur le deuil des proches. 


\section{BIBLIOGRAPHIE}

Bacque M.F., « Voir ou ne pas voir le corps du défunt ", Jusqu'à la mort accompagner la vie, 2015/2 ( $\left.N^{\circ} 121\right)$, p. 73-82. DOI : 10.3917/jalmalv.121.0073. URL : https://www.cairn.info/revuejusqu-a-la-mort-accompagner-la-vie-2015-2-page-73.htm.

Charlard L., 2020, Mesures Covid, Marseille versus Paris, Girondins contre Jacobins in : Figaro Vox, 28 septembre 2020, https://www.lefigaro.fr/vox/societe/mesures-covid-marseille-versusparis-girondins-contre-jacobins-20200928.

Cros M., 2015, Du sida à Ebola. Les rites de mort à fonction apotropaïque in L'Autre, 2015/3 vol.16, 263-274.

Epelboin A., 2020, Les épidémies peuvent entamer notre humanité, entretien, Sciences Humaines, 25 mars 2020, https://www.scienceshumaines.com/les-epidemies-peuvent-entamer-notrehumanite-entretien-avec-alain-epelboin_fr_42190.html

Journal officiel, Décret no 2020-384 du 1er avril 2020 complétant le décret no 2020-293 du 23 mars 2020 prescrivant les mesures générales nécessaires pour faire face à l'épidémie de covid-19 dans le cadre de l'état d'urgence sanitaire. Ministère des solidarités et de la santé.

Gasquet-Blanchard C., 2016, Ebola. Géographie d'une crise sanitaire. 1994-2005, P.U.R., 228 p.

Gasquet-Blanchard, C., 2015, « Ebola, géographie d'un virus. Enjeux socio-spatiaux en Afrique Centrale », L'Espace Politique [En ligne], 26 | 2015-2, mis en ligne le 22 juillet 2015, consulté le 30 septembre 2020. URL : http://journals.openedition.org/espacepolitique/3475 ; DOI : https:// doi.org/10.4000/espacepolitique.3475

Geoffard P.Y., 2020, Covid-19, les limites du Jacobinisme sanitaire in Les Echos, 2 septembre 2020. https://www.lesechos.fr/idees-debats/cercle/opinion-covid-19-les-limites-du-jacobinismesanitaire-1238608

Grimault V., 2020, « Tous égaux face à la pandémie ? La France du Covid-19 en 10 cartes », in Alternatives économiques, 6 avril.

Gueullette J.M., 2008, La toilette funéraire. Dernier des soins, premiers des rites in 2008/11, 409, 463-472 https://doi.org/10.3917/etu.095.0463

Lecompte Francis, 2020, Les pratiques funéraires face à la crise, entretien auprès de Pascale Trompette, Le Journal du CNRS, https://lejournal.cnrs.fr/articles/les-pratiques-funeraires-facea-la-crise.

HCSP, 2020, Avis relatif à la prise en charge du corps d'un patient décédé infecté par le coronavirus SARS-CoV-2, 18 février 2020, 6 p.

HCSP, 2020, Avis du 24 mars 2020 relatif à la prise en charge du corps d'un patient cas probable ou confirmé COVID-19, 9 p. Accessible sur https://www.hcsp.fr/Explore.cgi/ AvisRapportsDomaine?clefr $=786$

HCSP, 2020, Avis du 30 novembre 2020 relatif à la Prise en charge du corps d'une personne décédée et infectée par le coronavirus SARS-CoV-2 : actualisation des recommandations. Accessible sur : https://www.hcsp.fr/Explore.cgi/AvisRapportsDomaine?clefr=957

Kra F., Taverne B., MINIMEL F., Laborde-Balen G., Egrot M., 2020, L'anthropologie impliquée à l'hôpital en contexte d'épidémie de covid 19 pour accompagner les fins de vie et les décès hospitaliers, The Conversation, https://hal.archives-ouvertes.fr/hal-02956525/document. 
Levratto, N., Amdaoud M., Arcuri G., 2020 « Covid-19: analyse spatiale de l'influence des facteurs socio-économiques sur la prévalence et les conséquences de l'épidémie dans les départements français » Université Paris Nanterre, EconomiX, working paper, $\mathrm{n}^{\circ} 2020-4$.

OCDE, 2020, Communication from the Commission on the precautionary principle /* COM/ 2000/0001 final */Openness and Transparency - Pillars for Democracy, Trust and Progress.

OPECST, 2020a, « La crise du funéraire en situation de Covid-19: mort collective et rituels funéraires bouleversés », Sénat - République Française, 2 juillet 2020, 20 p. http:// www2.assemblee-nationale.fr/content/download/311629/3025735/version/1/file/ OPECST_2020_0027_note_rites_fun\%C3\%A9raires_covid19.pdf

OPECST, 2020b, « Les rites religieux face à l'épidémie de Covid-19 en France », Sénat - République Française, 2 juillet 2020, 38 p. http://www.senat.fr/fileadmin/Fichiers/Images/opecst/ quatre_pages/OPECST_2020_0028_note_cultes_covid19.pdf

Pérez S., et al. 2020, Comparative analysis of the perception of nuclear risk in two populations (expert/non-expert) in France. Energy Reports, 2020, 6, pp.2288-2298.

Pérez S., 2019, Etude AIR-SANTE en Pays de Martigues : apports de l'Intelligence Artificielle aux questions de santé environnementale, Rapport de Recherche, V1 octobre 2018, V2 juin 2019, 255 pages.

Renn O., 2015, Precaution and Ecological Risk, Reference Module in Earth Systems and Environmental Sciences, Elsevier, 2015

Ricci P., 2011, Special Issue on : Causal models in public policy. Dose-Response.

Ricci P. \& Sheng H-X., 2013, Benefits and Limitations of the Precautionary Principle. 10.1016/ B978-0-12-409548-9.01935-7.

Setbon M., 2004, Risques, sécurité sanitaire et processus de décision, Elsevier, 176p.

Sriwijitalai W., Wiwanitkit V., 2020, Corrigendum to "COVID-19 in forensic medicine unit personel: Observation from Thailand" [J Forensic Legal Med 72 May 2020, 101964]. Journal of Forensic and Legal Medicine,72,101967. https://doi.org/10.1016/j.jflm.2020.101967

T.M.C. ASSER INSTITUUT, 2011, Considerations on the application of the Precautionary Principle in the chemicals sector Final Report August 2011

Trompette P., Potier U., 2020, Le funéraire, « une autre première ligne », PUG, Collection le Virus de la Recherche, 5-9.

Vanier M., 2020, Une République des territoires à déconfiner ? in TELOS, 14 mai 2020 https:// www.telos-eu.com/fr/societe/une-republique-des-territoires-a-deconfiner.html

\section{NOTES}

1. Le 30 janvier 2020, l'Organisation Mondiale de la Santé (OMS) a déclaré la flambée du nouveau coronavirus (2019-nCOV) comme une Urgence de Santé Publique de Portée Internationale (USPPI), le 11 mars 2020, la maladie virale COVID-19 est qualifiée de pandémie. Fin août 2020180 pays étaient concernés https:// gisanddata.maps.arcgis.com/apps/opsdashboard/index.html\#/ bda7594740fd40299423467b48e9ecf6

2. Version initiale de cet article :11/09/2020. 
3. Des travaux en cours montrent que les personnes "anti-vaccins " sont aussi celles qui se disent "anti masques» dans le cadre de l'épidémie de Covid-19. https:// www.lemonde.fr/idees/article/2020/08/25/l-adhesion-aux-differentes-theories-ducomplot-en-est-un-trait-caracteristique-des-antimasques_6049841_3232.html

4. Chargé d'éclairer le gouvernement sur les mesures à prendre

5. Le nom de la maladie est COVID-19, pour COrona-VIrus Disease de 2019 ; le nom du virus lui-même étant SARS-CoV-2.

6. Veille anthropologique des maladies émergentes par Alain Epelboin; http:// www.cres-paca.org/r/257/covid-19-la-veille-du-cres/;

newsletter@eml.lequotidiendumedecin.fr ; https://www.santepubliquefrance.fr/ dossiers/coronavirus-covid-19/covid-19-etat-des-connaissances-et-veille-

documentaire ; https://bu.univ-amu.libguides.com/c.php?g=679422\&p=4843649

7. A la demande du Directeur général de la Direction Générale de la Santé (3 février 2020) un groupe d'expertise dédié au coronavirus a été mis en place au HCSP pour évaluer et émettre des recommandations. 84 saisines ont été transmises par la DGS dans les 7 premiers mois de l'épidémie cf. annexe de l'avis du 18/02/2020

8. https://doi.org/10.1016/j.jflm.2020.101967

9. Cf. le nota bene en fin d'article sur l'actualisation de ce deuxième avis

10. La mise en bière dite « immédiate » doit se faire dans un délai de 24 heures, elle est recommandée dans un certain nombre de maladies contagieuses fixées par arrêté comme par exemple : la grippe aviaire, la maladie de Creuztfeldt-jakob, le choléra, la peste, le SRAS

11. De plus, le fait de ne pas voir le visage du défunt rendrait difficile le processus de deuil (Bacqué, 2015)

12. https://www.francebleu.fr/infos/sante-sciences/coronavirus-un-syndicat-despompes-funebres-menace-de-faire-jouer-un-droit-de-retrait-1584468053

13. cf. par exemple un entrepôt vide réquisitionné et transformé en morgue à Mulhouse https://actu.fr/grand-est/mulhouse_68224/coronavirus-alsace-mulhouseentrepot-transforme-morgue_32842761.html>

14. https://www.ladepeche.fr/2020/04/10/fosses-communes-camions-frigorifiques-anew-york-des-scenes-dhorreur-pour-inhumer-les-victimes-du-coronavirus, 8841951.php

15. Voir également sur le statut et le ressenti des employés du funéraires, Trompette P., Potier U, 2020

16. Cluster, terme anglo-saxon qualifiant les «foyers d'infection» et une zone de circulation active du virus. Terme épidémiologique popularisé dans l'opinion publique pendant l'épidémie

17. Levratto, Amdaoud et Arcuri (2020) montrent également dans leur article que cette hétérogénéité est sociale dans la mesure où ils constatent que le taux d'hospitalisation, les taux de décès et de surmortalité sont d'autant plus élevés dans un département que celui-ci présente une forte densité, que les ouvriers représentent une part importante dans la population active et que les inégalités de revenu (mesurées par le rapport interdéciles) sont élevées (cité par Anota, 2020)

18. https://blogs.alternatives-economiques.fr/anota/2020/04/23/covid-19-1-epidemieest-la-plus-meurtriere-la-ou-les-inegalites-sont-les-plus-fortes 
19. Goubert Guillaume, «Le virus n'est pas Jacobin» https://www.la-croix.com/ Debats/Editos/Le-virus-nest-pas-jacobin-2020-04-20-1201090330

20. https://www.lesechos.fr/idees-debats/cercle/opinion-covid-19-les-limites-dujacobinisme-sanitaire-1238608

21. https://www.lefigaro.fr/vox/societe/mesures-covid-marseille-versus-parisgirondins-contre-jacobins-20200928

22. https://nicepresse.com/soutien-ordre-regional-des-medecins-professeur-raoult/

23. Le journal Libération en mai 2020 mentionne "l'adulation" dont bénéficie le Professeur Raoult de la part des marseillais malgré les controverses scientifiques https://www.liberation.fr/france/2020/05/26/a-marseille-raoult-est-un-che-guevarade-chez-nous_1789497. En juin 2020 le journal Le Parisien rend compte de l'engouement de la population en titrant «J'ai confiance en lui à $1000 \%$ : à Marseille Didier Raoult continue de séduire » https://www.leparisien.fr/societe/sante/au-coeurdu-laboratoire-du-professeur-raoult-23-06-2020-8340711.php

24. D'après un sondage Opinionway intitulé « Les Français et les risques de conflit " réalisé en février 2020, $57 \%$ des Français estiment que le niveau d'action le plus pertinent pour améliorer les choses en matière de santé est le niveau national, contre $11 \%$ pour le niveau local et $10 \%$ pour le niveau européen. Il en est de même pour les questions liées à la protection sociale, à l'emploi ou encore à l'éducation (p.31). https:// www.reseau-canope.fr/fileadmin/user_upload/Projets/CanoTech/Articles_CanoTech/ opinionway_decentralisation.pdf

25. On peut noter la forte opposition dès qu'il a été envisagé un déconfinement différencié de la population en fonction des âges.

26. https://www.lemonde.fr/planete/article/2020/08/22/de-nice-a-lille-la-france-seconvertit-au-port-du-masque_6049607_3244.html

\section{RÉSUMÉS}

La gestion de la crise sanitaire consécutive à la pandémie de la Covid-19 a mis en exergue la discordance entre un Etat centralisateur, en charge de la prévention de sa population, qui en situation sanitaire d'urgence applique des mesures uniformes sur l'ensemble du pays, et la population dont le vécu quotidien dans son territoire ne lui renvoie pas forcément la même lecture de l'évènement et des logiques d'action. Il nous parait intéressant de réfléchir au décalage qui peut exister entre une connaissance objective des phénomènes pouvant impacter la santé, telle qu'elle peut être mesurée par des scientifiques, ou des experts, sur un espace donné, et leurs représentations par les habitants, au sein de leur espace de vie en situation de pandémie virale. Que se passe-t-il, en fait à la rencontre du top down et du bottom up ? Comment s'articulent mesures de l'espace, principe de précaution, mesures de prévention, décisions politiques et perceptions de la population. Ces perceptions sont généralement fondées sur des représentations profanes de la maladie, de son étiologie, et sont issues de leur vécu quotidien dans un espace territorial donné, alors que la mesure des experts qui s'appuie sur des données quantifiées rend compte d'une dimension pouvant être considérée comme plus éloignée des préoccupations des 
populations, plus distante, plus froide. Cela est parfois renforcé par la méconnaissance de la part de la population des méthodes employées par les scientifiques. Cette différence entre mesure et perception d'une même situation peut générer entre les deux des sentiments d'incompréhension, d'inhumanité, surtout lorsque les discours scientifique ou politique se veulent plus objectifs et deviennent plus alarmistes que celui « attendu » ou perçu par les populations.

De plus, alors que les organismes et institutions publiques ont en charge le collectif, la population répond souvent par des stratégies individuelles. Se pose alors pour le décideur la question de la diversité des comportements, alors qu'il est à la recherche d'invariants, d'éléments suffisamment reproductibles qui seraient applicables en tous points du territoire.

Nous illustrerons cette approche à partir d'un exemple qui nous semble emblématique et qui concerne les mesures adoptées en matière de gestion du corps des personnes décédées pendant l'épidémie.

Par rapport au risque potentiel de transmission du virus nous mettrons en évidence les différences entre l'avis des scientifiques et des experts de limiter les interactions tout en conservant les dimensions humanistes des enterrements (avis du HCSP), la décision politique d'une mise en bière immédiate (arrêté du 2 avril 2020), et l'incompréhension des familles de ne pouvoir se déplacer et pratiquer les rites funéraires habituels. Nous discuterons donc de l'application des décisions prises entre mesures et perceptions du risque, entre mesures nationales et perceptions locales.

Nous montrerons comment tout un ensemble de décisions prises au niveau strictement national a conduit à des processus identitaires exacerbés entre certaines régions et la capitale s'appuyant sur une opposition plutôt qu'une complémentarité entre mesures et perceptions.

INDEX

Mots-clés : Covid-19, crise, gestion de crise, principe de précaution, rites funéraires

Keywords : Covid19, crisis, crisis management, precautionary principle, funeral rites

\section{AUTEURS}

\section{SANDRA PÉREZ}

Géographe de la santé, Université Côte d'Azur, UMR 7300, ESPACE, Nice, France

\section{NICOLE VERNAZZA-LICHT}

Anthropologue de la santé, UMR 7300, ESPACE, Nice, France

\section{DANIEL BLEY}

Anthropologue biologiste, UMR 7300, ESPACE, Université d'Aix-Marseille, France 